\title{
Impact of video clinics in the management of fracture nasal bones in COVID times*
}

\section{Ved Narang, Vandana Eswarappa, Nick Calder}

Department of ENT, University Hospital Monklands, Airdrie, Scotland, UK
Rhinology Online, Vol 4: 127 - 130, 2021

http://doi.org/10.4193/RHINOL/21.026

*Received for publication:

May 26, 2021

Accepted: July 27, 2021

Published: August 13, 2021

\begin{abstract}
Background:The COVID -19 pandemic created a panic situation where patient interaction with the other patients and health care staff had to be restricted to avoid spreading the disease. We planned an innovative strategy to restrict the inflow of patients to those who may need nasal bone manipulation by using Video Clinics (VC).

Methods: All consecutive patients presenting to three units of Accident \& Emergency (A \& E) NHS sites of our trust with suspicion of fractured nasal bones were included in the study group for a period of three months. The impact of VC was studied by calculating the percentage of patients who could be discharged without a recall to the hospital from those attending it, the percentage who finally needed a fracture reduction, and the satisfaction scores of patients with VC.
\end{abstract}

Results: Forty-two patients were offered appointments in VC. Thirty-one could attend VC, out of which thirteen (41.9\%) were discharged without a recall. Twenty-three patients were recalled for F2F (Face2Face) clinic, out of which twelve (28.5\% of 42) required fracture reduction, and 11 were discharged to home. Ten patients underwent fracture reduction under local anaesthesia, and two were booked for Septorhinoplasty later. Twenty-seven (87.1\%) patients expressed satisfaction with VC, two (6.4\%) were lost to follow-up, and the remaining two (6.4\%) were not satisfied.

Conclusion: VC could effectively filter $41.9 \%$ of patients who did not need surgical intervention from the comfort of their homes or workplace by using the available multimedia facility without compromising outcomes and satisfaction scores. The satisfaction score was $87.1 \%$ with VC. The clinics helped prevent potential coronavirus exposure by staying safe, and they are recommended in emergencies like COVID -19.

Key words: accident and emergency, COVID-19, SARS-CoV-2, face to face clinic, fracture nasal bones, nasal bone manipulation, video iClinic

\section{Introduction}

We describe an innovative approach in managing patients referred for nasal bone trauma to the NHS system during the COVID pandemic time. The nose is known to house high concentration of corona virus in infected individuals ${ }^{(1)}$.

We ran a nasal fracture video clinic (VC) to minimize the risk of transmission of the virus to the public and hospital staff.

Since a significant number of patients in our practice with nasal bone trauma do not require surgical intervention, we planned to identify these patients through a video clinic. In our study we were successful in identifying nearly $42 \%$ of patients who could be discharged with high patient satisfaction scores thus saving time and revenue. In a situation where it may be difficult to perform a clinical examination, like current COVID pandemic, this model can be used as an effective alternative emergency diagnostic solution. 


\section{Materials and Methods}

All patients presenting with suspected closed fracture nasal bones were included in the prospective observational study for a period of three months starting 15th July 2020. The Accident \& Emergency (A\&E's) of three acute University Hospital sites of our Health Board were circulated the following new instructions:

- To assess patients as per usual protocols and exclude septal hematoma in A\&E by the health care professional and a medico legal record was created if required. Patients with septal hematoma to be managed by the ENT on call team.

- All patients to be booked for a VC in 7 to 14 days of injury. The VC was held once per week and the patients were requested to connect using a $\mathrm{QR}$ code or a web address. These were printed and mailed to their current residential address.

The shape of the nose was examined in frontal and profile views by the lead author in VC. The nasal passage was assessed by requesting patients to gently press the ala against the septum and breathe from the other side. An attempt was made to compare the photograph of the face prior to injury with the present shape of the nose, which usually was the case.

All the patients with straight noses and normal breathing were discharged from VC. Patients with apparent deviations and or difficulty in breathing were booked for a Face-to-Face clinic appointment on the next working day.

Nasal bone manipulation (NBM) under Local Anesthesia (LA) was performed in face 2 face clinic for patients who could tolerate it at the same time and the rest were scheduled under General Anesthesia (GA) at a later time point. All patients attending VC were contacted after 4 to 6 weeks via telephone, to gather their feedback to VC and reconfirm their outcomes.

\section{Results}

A total of 42 patients ( 23 males and 19 females) with complains of nasal bone injury were referred from A\&E to VC. Thirteen (30.95\%) were children less than 18 years of age. The male to female ratio was 1.21:1.

Seventeen adults were injured because of spontaneous fall and the remaining twelve sustained injury due to accident, assault and sports. Children were more often injured more oftenbecause of assault and sports, five in each category and the remaining three were due to spontaneous fall.

Thirty-one patients (74\%) out of forty-two could attend the video clinic. Of patients failing to attend VC, three could be reached on their mobile phones, three were lost to follow up and five were booked directly for the Face-to-Face clinic (Table 1).
Table 1. An analysis of patients offered appointment in video clinic and their actual attendance.

number

Total No. of patients offered Video Clinic (VC) appointments

$\mathrm{N}=42$

Patients who could attend VC

31

Patients failed to attend VC due to technical reasons and old age.

(Hence offered direct F2F appointment)

Patients failed to attend VC, answered the phone and they were happy to be discharged.

Patients failed to attend VC and failed to answer the phone (Lost to follow up)

Direct F2F (Face-to-Face) clinic appointments due to public holidays

Table 2. Impact of video clinic in managing nasal bone trauma patients and an analysis of Face-to-Face clinic appointments *NBM (Nasal Bone Manipulation).

\begin{tabular}{|lc|}
\hline & number \\
\hline Total number of patients attending VC (N) & 31 \\
\hline Discharged from VC (41.9\% of N) & 13 \\
\hline Appointment from VC to Face-to-Face clinic (58.1\% of N) & 18 \\
\hline Direct Appointments to Face-to-Face clinic & 5 \\
\hline Total Attending Face-to-Face clinic & 23 \\
\hline Patients discharged from Face-to-Face clinic & 11 \\
\hline Patients needing NBM* under LA / GA & 12 \\
\hline
\end{tabular}

Table 3. Satisfaction scores with video clinic.

\begin{tabular}{|lcc|}
\hline Satisfaction Score & $\begin{array}{c}\text { Number of } \\
\text { Patients }\end{array}$ & $\begin{array}{c}\% \text { of } \\
\text { patients }\end{array}$ \\
\hline Very Satisfied & 25 & $80.6 \%$ \\
\hline Satisfied & 2 & $6.4 \%$ \\
\hline Dissatisfied & 2 & $6.4 \%$ \\
\hline No Information & 2 & $6.4 \%$ \\
\hline Total & 31 & $100 \%$ \\
\hline
\end{tabular}

Thirteen (42\%) out of thirty-one patients attending VC were discharged and eighteen were booked for face 2 face clinic appointment (Table 2). Twenty-three (54.8\%) out of 42 patients attended the F2F clinic. Eighteen were booked from VC and another five were booked directly. Eleven patients were discharged after VC and twelve (28.5\%) required NBM as final outcome. Ten patients (23.8\%) had NBM performed under LA and two (4.8\%) were scheduled for Septorhinoplasty under GA later. 
Twenty-five (80.6\%) out of 31 patients attending VC were very satisfied and two (6.4\%) were satisfied (Table 3). Two patients (6.4\%) expressed dissatisfaction and two (6.4\%) were lost to follow up as they failed to answer telephone calls.

\section{Discussion}

The institutional and individual behavior in society greatly affects the spread of the infectious diseases ${ }^{(2)}$. The strategy in managing fracture nasal bones was to keep personal interaction to a minimum without compromising the patient's interest.

The focus in VC was on the nasal shape and breathing. Dedicated fracture clinics are seen to reduce time to assessment and management ${ }^{(3)}$, but the authors could not trace any literature on VC. Merging the techniques of dedicated clinic and use of video seemed highly relevant in pandemic times and we wanted to share our results for the wider benefit.

No radiological procedure was performed to diagnose closed fracture nasal bones, which are generally simple in nature and clinical assessment is the key ${ }^{(4)}$. One-to-two-week interval to VC helped oedema to subside and offer NBM under 3 weeks ${ }^{(5)}$.

Nasal bone fracture is the most common facial bone injury accounting for $39-45 \%$ of all facial fractures ${ }^{(6)}$. The male to female ratio can vary from 2:1 ${ }^{(6,7)}$ to a ratio of 72:28 ${ }^{(8)}$. Our figures of 1.21:1 for M: F ratio; are different from other studies where males were predominantly affected.

The most common mode of injury was spontaneous fall in adults accounting for seventeen (58.6\%) out of 29 patients. This is different from previous studies where assault was noted as the most common cause. Forty three percent patients sustained fracture due to assault followed by sports injuries in $23.8 \%$ and fall in $21.3 \%$ (7) in a large study of 483 patients. The differences in the sex ratio and the cause for fracture nasal bone could be due to people spending more time indoors, limited personal interaction and reduced traffic activity.

Out of the 42 patients assigned for VC, only thirty-one (73.8\%) could connect. Children were usually accompanied by an adult. Thirteen (41.9\%) out of thirty-one patients booked were discharged at the first instance. Since this a new concept, we are unable to compare and contrast these figures with any previous literature.

The discharged patients were happy with the shape of their nose and breathing and assessing surgeon was also satisfied. They gained the maximum from VC as they avoided a return visit to the hospital, escaped proximity to other patients and attended appointment from the privacy of their homes or workplace.
Three patients (7.1\%) were happy to self-discharge themselves. They were contacted via telephone as they failed to connect on VC. They were happy with their noses and we accepted their decisions. Three patients (7.1\%) failed to connect and also failed to answer telephonic calls; hence, they were lost to follow up.

In any circumstances where the patients could not be reviewed within two weeks of injury, they were booked directly for Faceto-Face clinic, which happened in five (11.9\%) of our patients. Two patients had problems with their phone cameras, one elderly patient found it challenging to connect and the remaining two missed their appointment due to a public holiday. Missed appointments due to public holidays can be addressed by arranging the clinic twice a week, as our dedicated clinic was limited to once a week.

Eighteen out of 31 patients attending VC had to be called again to the Face-to-Face clinic on the next working day due to apparent nasal deviation or suspected reduced nasal airflow. They were joined by five patients who were booked directly thus forming a group of 23. Two patients in this group insisted to be seen in clinic before discharge.

Twelve patients out of twenty-three booked in Face-to-Face clinic required further management by NBM. Eleven patients were discharged after evaluation as needed no further intervention. We expect the ratio of patients requiring NBM to discharge without intervention, to improve in future as the idea of VC gains acceptance of the people and clinicians gain more experience.

Ten patients had their NBM performed under LA at the time of clinic appointment. Two patients were scheduled for Septorhinoplasty (SPR) later, as one tested positive for COVID-19 on the day of scheduled NBM under GA and the other missed the appointment twice and hence was unsuitable for NBM.

NBM at an optimal time is key to successful management. Testing COVID-19 positive on the morning of surgery without any symptoms, points to presence of asymptomatic carriers and deferring surgery helped prevent further spread of the virus ${ }^{\left({ }^{9}\right.}$.

\section{Satisfaction score}

The patients attending VC were contacted after 4 to 6 weeks of appointment via telephone to collect their feedbacks and ensure satisfaction with their outcomes. Twenty-nine patients (93.5\%) could be contacted out of thirty-one, and two (6.4\%) failed to answer the telephone call, hence remained untraceable.

Twenty-five patients (80.6\%) were very satisfied with video consultation and two (6.4\%) were satisfied. The very satisfied and satisfied patients were of the opinion that it saved them 
time, money and driving efforts. Three of the thirteen patients attended VC from their workplace, thus saving them half a day's salary.

Two patients (6.4\%) expressed dissatisfaction for the reasons of loss of personal touch. It is very encouraging to see satisfaction levels of $87 \%$ with a new idea and we hope to improve these scores as people develop more confidence in VC.

\section{Conclusions}

In our opinion, management of suspected fracture nasal bones can be helped in a safe and an effective way by using Video Consultations. We recommend this process as an alternative emergency diagnostic solution in situations like the current COVID-19 pandemic. $42 \%$ patients with an undisplaced fracture could be discharged from $\mathrm{VC}$ without a recall. A high satisfaction rate of $87 \%$ with the VC consultation process is very encouraging.

The appointments for VC can be easily built into the existing system by using the existing multi-media facility present at most of the hospitals without extra costs.

\section{Abbreviations}

A\&E, Accident and Emergency; GA, General Anesthesia; LA, Local Anesthesia; NBM, Nasal Bone Manipulation; SRP, Septorhinoplasty; VC, Video Clinic.

\section{Authorship contribution}

VN: Concept \& designing of the project, Data collection and Analysis, Clinical examination of patients in Video clinic, Writing of the article, Review and accepting the final report. VE: Designing the project, Data Collection and Analysis, Reviewing and accepting the final report. NC: Concept \& Designing of the Project, Critical inputs while writing the report, Reviewing and accepting the final report.

\section{Acknowledgments}

None.

\section{Funding}

Not applicable.

\section{Ethics approval and consent to participate}

Not applicable.

\section{Consent for publication}

Not applicable.

\section{Availability of data and materials}

Not applicable.

\section{Conflict of interest}

The authors have no conflicts of interest to declare.

\section{References}

1. Wang W, Xu Y, Gao R, Lu R, Han K, Wu G, Tan W. Detection of SARS-COV-2 in Different Types of Clinical Specimens. JAMA. 2020 May 12;323(18):1843-1844

2. Laxminarayan, R \& Malani, A. Economics of infectious diseases. Oxf. Handb. Health Econ.https://doi.org/10.1093/oxfordhb/9780199238828.013.0009(2012).

3. Pinto R, Wright $R$, Ghosh S. Nasal fractures: a dedicated clinic providing reduction under local anaesthesia improves time to manipulation. Ann R Coll Surg Engl. 2020 Jul;102(6):418-421.

4. Sharp JF, Denholm S. Routine X-rays in nasal trauma: the influence of audit on clinical practice. J R Soc Med. 1994 Mar;87(3):153-4.

5. Hoffmann JF. An Algorithm for the Initial Management of Nasal Trauma. Facial Plast
Surg. 2015 Jun;31(3):183-93.

6. Hussain K, Wijetunge DB, Grubnic S, Jackson IT. A comprehensive analysis of craniofacial trauma. J Trauma. 1994 Jan;36(1):34-47.

7. Renkonen S, Vehmanen S, Mäkitie A Blomgren K. Nasal bone fractures are successfully managed under local anaesthesia - experience on 483 patients. Clin Otolaryngol. 2016 Feb;41(1):79-82.

8. Basheeth N, Donnelly M, David S, Munish S. Acute nasal fracture management: A prospective study and literature review. Laryngoscope. 2015 Dec;125(12):2677-84.

9. Zhou F, Li J, Lu M, Ma L, Pan Y, Liu X, Zhu X, Hu C, Wu S, Chen L, Wang Y, Wei Y, Li Y, Xu H, Wang $X$, Cai L. Tracing asymptomatic SARSCoV-2 carriers among 3674 hospital staff: a cross-sectional survey. EClinicalMedicine. 2020 Sep;26:100510.

\author{
Mr. Ved Narang \\ Department of ENT \\ University Hospital Monklands \\ NHS Lanarkshire \\ Airdrie, ML60JS \\ Scotland, UK \\ Tel: (Secretary) - 00441236742332 \\ E-mail: vednarang@yahoo.com \\ VedPrakash.Narang@Lanarkshire. \\ scot.nhs.uk
}

ISSN: 2589-5613 / 일 2021 The Author(s). This work is licensed under a Creative Commons Attribution 4.0 International License. The images or other third party material in this article are included in the article's Creative Commons license, unless indicated otherwise in the credit line; if the material is not included under the Creative Commons license, users will need to obtain permission from the license holder to reproduce the material. To view a copy of this license, visit http://creativecommons.org/ licenses/by/4.0/ 